\title{
In-situ visualization of stress-dependent bulk magnetic domain formation by neutron grating interferometry
}

\author{
B. Betz, ${ }^{1,2}$ P. Rauscher, ${ }^{3}$ R. P. Harti, ${ }^{1}$ R. Schäfer, ${ }^{4}$ H. Van Swygenhoven, ${ }^{2,5}$ A. Kaestner, ${ }^{1}$ \\ J. Hovind, ${ }^{1}$ E. Lehmann, ${ }^{1}$ and C. Grünzweig ${ }^{1}$ \\ ${ }^{1}$ Paul Scherrer Institut, Laboratory for Neutron Scattering and Imaging, CH-5232 Villigen, Switzerland \\ ${ }^{2}$ École Polytechnique Fédérale de Lausanne, NXMM Laboratory, IMX, CH-1015 Lausanne, Switzerland \\ ${ }^{3}$ Fraunhofer IWS Dresden, Laser Ablation and Cutting, D-01277 Dresden, Germany \\ ${ }^{4}$ Leibniz Institute for Solid State and Materials Research (IFW) Dresden, Helmholzstr.20, D-01069 Dresden, \\ Germany and Institute for Materials Science, University of Technology, D-01069 Dresden, Germany \\ ${ }^{5}$ Paul Scherrer Institut, Photons for Engineering and Manufacturing, CH-5232 Villigen, Switzerland
}

(Received 18 November 2015; accepted 17 December 2015; published online 5 January 2016)

\begin{abstract}
The performance and degree of efficiency of industrial transformers are directly influenced by the magnetic properties of high-permeability steel laminations (HPSLs). Industrial transformer cores are built of stacks of single HPSLs. While the insulating coating on each HPSL reduces eddy-current losses in the transformer core, the coating also induces favorable inter-granular tensile stresses that significantly influence the underlying magnetic domain structure. Here, we show that the neutron dark-field image can be used to analyze the influence of the coating on the volume and supplementary surface magnetic domain structures. To visualize the stress effect of the coating on the bulk domain formation, we used an uncoated HPSL and stepwise increased the applied external tensile stress up to $20 \mathrm{MPa}$. We imaged the domain configuration of the intermediate stress states and were able to reproduce the original domain structure of the coated state. Furthermore, we were able to visualize how the applied stresses lead to a refinement of the volume domain structure and the suppression and reoccurrence of supplementary domains. (C) 2016 AIP Publishing LLC. [http://dx.doi.org/10.1063/1.4939196]
\end{abstract}

High-permeability steel laminations (HPSLs) are highly anisotropic grain-oriented electrical steels having a sharp (110) [001]-texture, the so-called Goss-texture. ${ }^{1}$ This texture leads to desired magnetic properties for use as transformer core material. $^{2-5}$ A typical HPSL is between $0.23 \mathrm{~mm}$ and $0.35 \mathrm{~mm}$ thick and is coated by a glassy magnesium silicate layer. This insulating coating reduces core losses in stacked transformer cores. The coating also has significant impact on the magnetic domain structure of the HPSL, which determines the performance of the transformer. ${ }^{5-9}$

Most commonly, HPSLs are investigated and characterized by inductive $B$ - $H$-hysteresis-measurements devices. This technique reveals global magnetic properties such as magnetic hysteresis, saturation, and losses. ${ }^{10}$ Locally resolved information about the underlying, determining domain structure cannot be obtained by this method. The surface domain structure is predominantly studied with Kerrmicroscopy investigations. ${ }^{11}$ The limited information depth of $20 \mathrm{~nm}$ only allows for indirect interpretation of the internal volume domain structures. Furthermore, the field of view is limited to a few millimeters. Perhaps, the biggest disadvantage of Kerr microscopy is that the coating must first be removed from the HPSL. To overcome these drawbacks, we conducted neutron grating interferometry (nGI) experiments. ${ }^{12}$ The neutron dark-field image (DFI) allows for the visualization of the bulk magnetic domain structure in two $^{13-15}$ and three ${ }^{16}$ dimensions. The nGI technique is established as a routine method in neutron imaging and used at facilities around the world. ${ }^{12,17-19}$ Here, we report on the localized visualization of the impact of the insulating coating on the magnetic domain structure for both volume and surface domain structures. We performed nGI experiments on the in-situ visualization of the magnetic domain response for externally applied tensile stresses in an uncoated HPSL.

The nGI experiments were carried out at the Swiss Spallation Neutron Source (SINQ) using the cold neutron imaging facility ICON. ${ }^{20}$ The setup used for the measurements is schematically shown in Fig. 1. An absorbing source grating, $\mathrm{G}_{0}$ (periodicity: $\mathrm{p}_{0}=1076 \mu \mathrm{m}$ ), is placed in a monochromatic neutron beam with a wavelength of $4.1 \AA(\Delta \lambda / \lambda \approx 15 \%)$ provided by a velocity selector. The source grating produces an array of periodic line sources that provide sufficiently high spatial coherence when illuminated with a large centimeter-sized

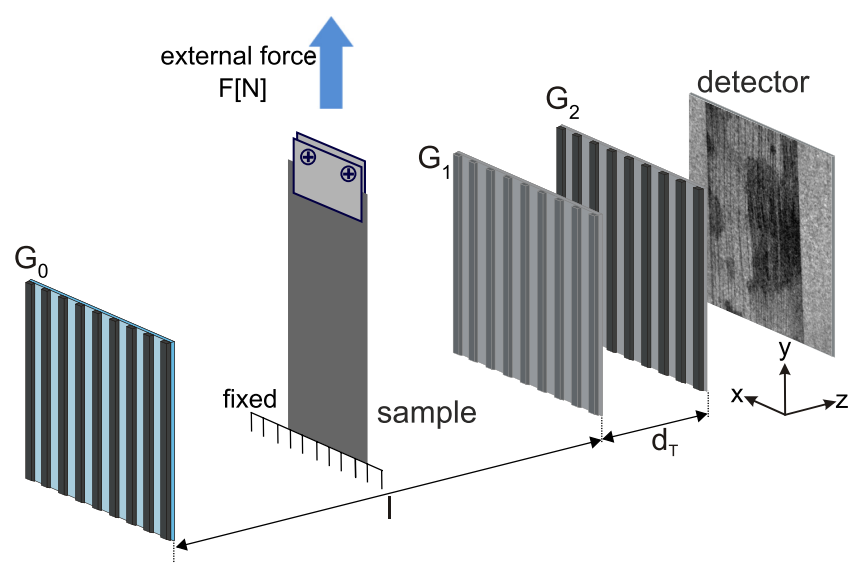

FIG. 1. Schematic drawing of neutron grating interferometer for the in-situ visualization of the magnetic domain response for externally applied tensile stresses. The source grating, $\mathrm{G}_{0}$, is placed at distance 1 in front of the phase grating, $\mathrm{G}_{1}$. The analyzer grating, $\mathrm{G}_{2}$, is located at the Talbot distance $\mathrm{d}_{\mathrm{t}}$ behind $\mathrm{G}_{1}$. The HPSL is mounted in a uni-axial tensile loading machine directly in front of $\mathrm{G}_{1}$. Images are formed by a conventional neutron detection system based on a scintillator screen and a digital camera. 
neutron beam. After a distance of $l=5.23 \mathrm{~m}$, a second grating (phase grating), $\mathrm{G}_{1}\left(\mathrm{p}_{1}=7.97 \mu \mathrm{m}\right)$, is placed. The phase grating introduces a phase modulation onto the incoming neutron beam, which is afterwards transferred into an intensity oscillation at distance $d_{T}$ after $G_{1}$, as a consequence of the Talbot effect. ${ }^{21}$ The third grating $\mathrm{G}_{2}\left(\mathrm{p}_{2}=4 \mu \mathrm{m}\right)$ analyzes this intensity pattern in combination with a state-of-the-art neutron imaging detection system. The images were recorded using a $100 \mu \mathrm{m}$ thick ${ }^{6} \mathrm{LiF} / \mathrm{ZnS}$ scintillator screen and a digital camera [Andor NEO sCMOS, $2160 \times 2560$ pixels, pixel size: $6.5 \mu \mathrm{m}$ ]. The effective spatial resolution of $70 \mu \mathrm{m}$ was determined by intrinsic blurring of the scintillation screen $^{22}$ and penumbra blurring caused by the sample to detector distance of $3 \mathrm{~cm}$. The HPSL sample is mounted in a uniaxial tensile loading machine which is placed as close as possible in front of $G_{1}$. The applied force is measured by a load cell [Soemer 614] with an accuracy of $0.5 \mathrm{~N}$.

In Fig. 2(a), a conventional transmission image (TI) of a coated HPSL is shown. The $300 \mu \mathrm{m}$ thick HPSL, which is an iron alloy with approximately $4 \%$ silicon, provides a weak but homogeneous contrast. In contrast to the attenuation based TI, the DFI is related to multiple refraction of unpolarized neutrons at magnetic domain walls. ${ }^{13-15}$ Further information about the contrast origin and data processing can be found in Ref. 13. The corresponding DFI of the coated HPSL is shown in Fig. 2(b). A pronounced heterogeneous contrast with sharp features is observed. The vertical black line patterns represent elongated magnetic volume domains, while the lines themselves are the domain walls. It is known ${ }^{5}$ that the magnetization of the volume domains in HPSLs points along the magnetic easy axis. The magnetic easy axis is the [001]-direction of the crystalline structure and points along the vertical axis in the DFI. These volume domains are found with a domain wall distance larger than the detector resolution. They are consequently visualized individually. ${ }^{13,14}$ Furthermore, a circular area with a decreased DFI signal is found in lower right part in the DFI. This is a misoriented grain where supplementary domains are generated to reduce magnetic stray fields. ${ }^{5}$ These supplementary domains, with a domain wall distance smaller than the detector resolution, contribute to an average degradation of the DFI signal. This DFI signal reduction is interpreted as a higher relative density of domain walls. ${ }^{15}$

The same HPSL in its uncoated state (procedure for the uncoating is described in Ref. 23) is shown in Fig. 2(c). Here, the DFI reveals qualitatively similar features but three noteworthy differences occur: (i) Grain boundaries become more visibly pronounced because some main domains are interrupted at the boundary. Additionally, small grain boundary domains are created at these boundaries (visible in the upper part of the DFI). (ii) The volume domain structure shows an increased domain width, clearly visible on the left side of the misoriented circular grain. (iii) Most notably, parts of the supplementary domains inside the circular grain vanish. It is known that the protective coating applies planar tensile stresses on the HPSL. They act as effective uniaxial tensile stresses in the rolling direction due to the sharp Goss-texture. These stresses, in combination with the inverse magneto-strictive effect, favor the [001]-direction compared to the transversal magnetic easy axis of the material. This leads to a suppression of supplementary domains. ${ }^{5}$ Accordingly, a removal of the coating implies a removal of tensile stresses. Consequently, the stress removal explains the occurrence of the supplementary domains at the grain boundaries in Fig. 2(c). The wider volume domain structure is also explained by the presence of the supplementary domains in the uncoated state. In the coated state, the magnetic stray fields are reduced by a refined volume domain structure. In the uncoated state, the stray fields can be compensated by the formation of supplementary domains, allowing for a wider volume domain structure. The supplementary domains in the circular grain being not suppressed in the coated state in Fig. 2(b) can be explained by a large misorientation of this grain. The heterogeneous reduction in contrast in this area after the coating is removed is an unexpected behavior. The reason for its occurrence remains unclear and does not fit into existing magnetic models.

As seen in Fig. 2, the removal of the coating significantly changes the underlying magnetic domain structure. This is seen by comparing the domain structures of the initial (uncoated) and final (coated) states. To analyze the stress dependent magnetic domain formation in more detail, we stepwise applied external mechanical forces. Starting from the uncoated stress-free state, we investigate the transition via the intermediate stress states up to the reoccurrence of the domain structure of the final coated state. The experimental results of the stress-dependent domain formation of the uncoated HPSL are shown in Fig. 3.

In our experimental conditions, the externally applied force is completely transferred into stress since no deformation of the HPSL is observed. In Ref. 7, it is shown that typical values of applied stresses are in the range of up to (a) Transmission image

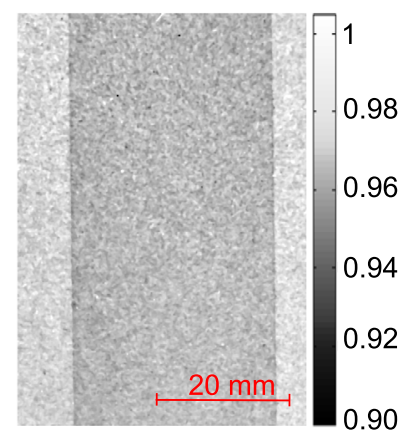

(b) Dark-field image (coated)

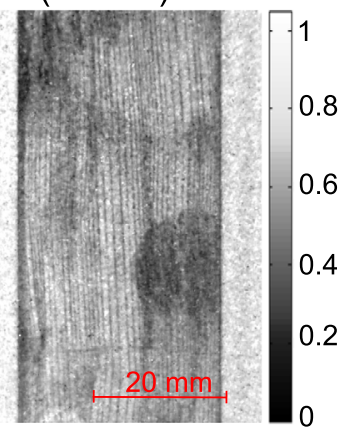

(c) Dark-field image (uncoated)

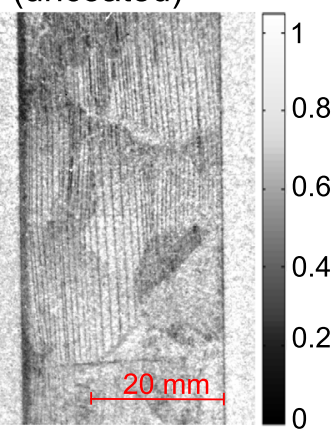

FIG. 2. (a) TI of the coated HPSL showing a weak and homogeneous contrast. (b) DFI of the same coated HPSL showing a pronounced contrast with dedicated features. Dark vertical lines represent magnetic domains walls, and dark areas visualize the existence of supplementary domains. (c) DFI of the same HPSL in its uncoated state. The volume domain as well as the supplementary domain structure has changed. 
(a) $0 \mathrm{MPa}$ uncoated

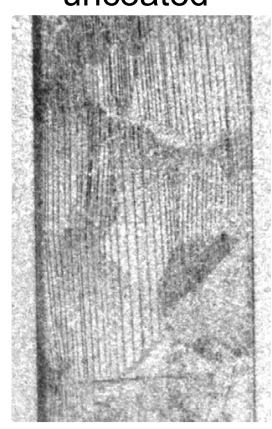

(d) $7.5 \mathrm{MPa}$ uncoated

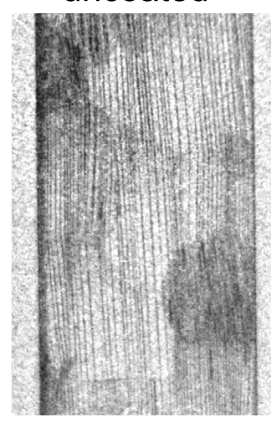

(b) $1 \mathrm{MPa}$ uncoated

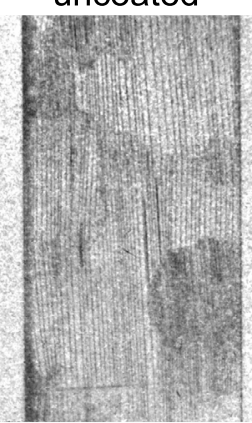

(e) $10 \mathrm{MPa}$ uncoated

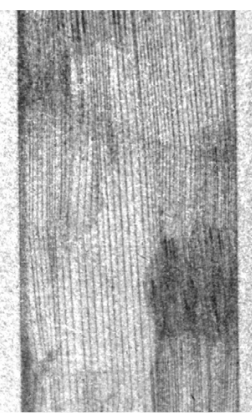

(c) $2.5 \mathrm{MPa}$ uncoated

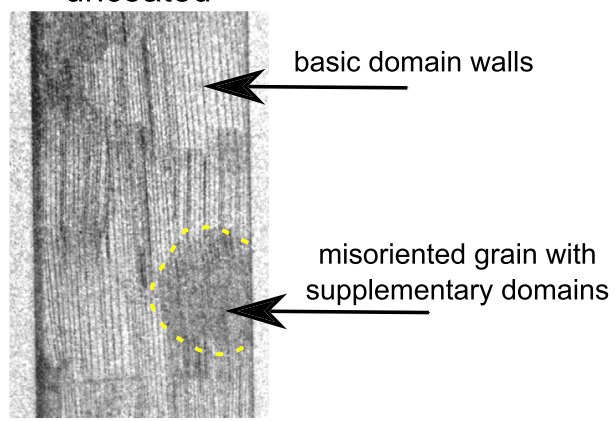

(f) $20 \mathrm{MPa}$ uncoated

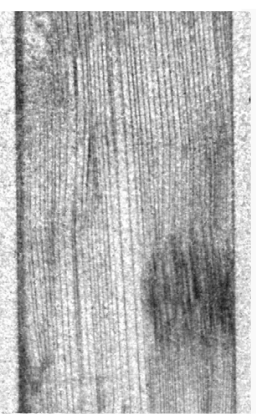

(g) $0 \mathrm{MPa}$ coated

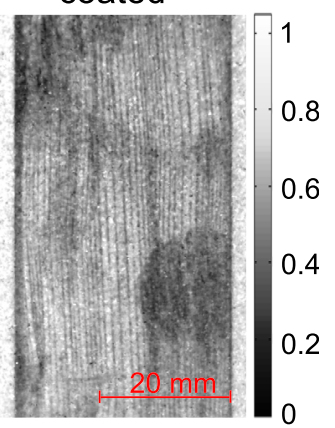

FIG. 3. (a) Uncoated HPSL without externally applied stress. (b)-(f) Increasing applied stresses up to $20 \mathrm{MPa}$ lead to a refinement of the volume domain structure, the suppression of grain boundary domains at grain boundaries, and a reoccurrence of the domain structures in the misoriented grain. (g) Coated HPSL without applied stress. several tenths MPa. The cross section of the HPSL is $0.3 \mathrm{~mm} \times 30 \mathrm{~mm}=9 \mathrm{~mm}^{2}$. To realize an applied stress of $\sigma=10 \mathrm{MPa}$, a force of $\mathrm{F}=90 \mathrm{~N}$ is needed. The maximum applied stress in our experiments was $20 \mathrm{MPa}$. This is far below a plastic deformation of the HPSL, since the yield strength of a HPSL is larger than $300 \mathrm{MPa} ;{ }^{24}$ hence, the conditions remain within the elastic regime.

In Fig. 3(a), the DFI of the uncoated HPSL without applied stress $(\sigma=0 \mathrm{MPa})$ is shown. It serves as both the initial and reference point for the comparison of the domain structure of the following different stress states. Fig. 3(b) shows the HPSL with $\sigma=1 \mathrm{MPa}$ applied stress. Already applying this small stress leads to a refinement of the volume domain structure. This can be clearly seen on the left side of the misoriented grain. Increasing the stress to 2.5 MPa-Fig. 3(c), 7.5 MPa-Fig. 3(d), $10 \mathrm{MPa}$-Fig. 3(e), and $20 \mathrm{MPa}$-Fig. 3(f) leads to further mutations of the volume and the supplementary domain structure. The changes in the volume structure do not manifest in a further refinement. Changes are clearly visible, but the average domain wall spacing does not show a trend and is roughly constant. It can be stated that the refinement process of the volume domain structure is predominantly happening when small stresses are applied. This can be seen in comparison to the coated state in Fig. 3(g). It can also be seen that as applied stress is increased, the visibility of the grain boundaries is reduced by the grain boundary domain density being decreased here. The grain boundaries are nearly invisible in the DFI with a stress of $20 \mathrm{MPa}$ Fig. 3(f) similar to that observed in the coated state in Fig. 3(g). Most notably, the supplementary domain structure, together with the underlying volume domain structure in the misoriented grain, reappears at an applied stress of only $\sigma=1 \mathrm{MPa}$ as shown in Fig. 3(b).
The application of increasing stresses leads to a more pronounced contrast of the misoriented grain in the corresponding DFI. The analysis of this effect is better illustrated in Fig. 4. The average dark-field values in the misoriented grain (green circle in the inset) are plotted versus the applied stresses. The red line in Fig. 4 represents the final DFI value of the coated HPSL. The grain in the uncoated HPSL in the stress-free state has a DFI value of 0.74 . The DFI value decreases as applied stress gradually increases. The reproduction of the initial DFI value from the coated HPSL is achieved with an applied stress of $20 \mathrm{MPa}$. Because the average DFI signal is a measure of the relative domain wall density, the magnetic domain structure in the coated state can be

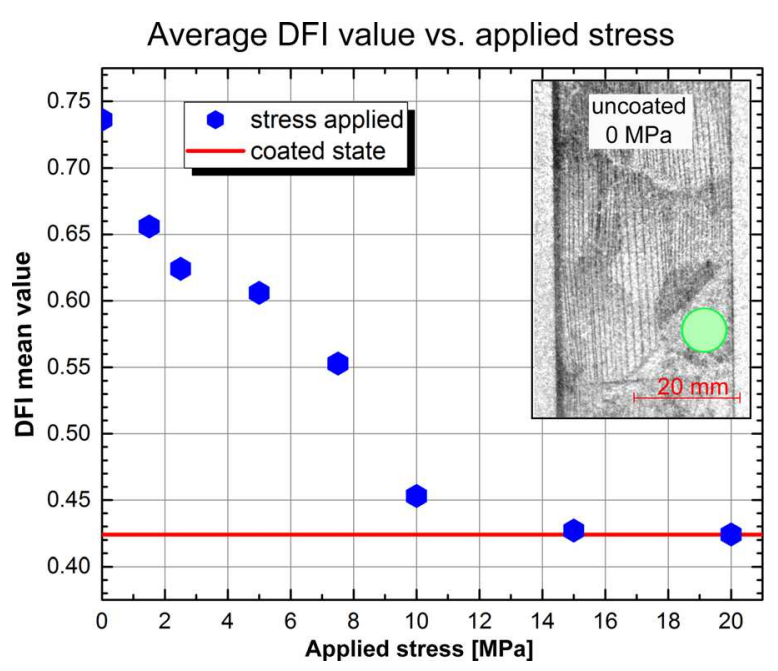

FIG. 4. Stress-dependent supplementary domain formation in the misoriented grain (area marked by the green circle in the inset). The DFI value for the coated state (red line) is reproduced at $20 \mathrm{MPa}$. 
reproduced to a similar degree in the misoriented grain. Interestingly, the supplementary domain structure is reproduced at significantly higher stresses than those required for the refinement of the volume domain structure.

In this article, we have shown how the neutron grating interferometry technique can be used for the in-situ visualization of stress-dependent volume and supplementary magnetic domain formation of a high-permeability steel lamination. We investigated the impact of removing the HPSL coating (and subsequent elimination of stress) on changes in the underlying domain structures. In particular, we analyzed the stress-dependent recovery of the domain structures under applied external forces. Both volume and supplementary structures were reproduced. Volume domains were reproduced at small stresses, whereas the supplementary domain structure in the misoriented grain was recovered at maximum applied stress values. The DFI findings presented here have the potential to further improve the properties of HPSLs and to improve existing approaches in the field of descriptive models for bulk macro-magnetic phenomena.

${ }^{1}$ N. P. Goss, U.S. patent 1,965,559 (3 July 1934.

${ }^{2}$ S. Taguchi, T. Yamamoto, and A. Sakakura., "New grain-oriented silicon steel with high permeability orientcore HI-B," IEEE Trans. Magn. 10, 123-127 (1974).

${ }^{3}$ S. Mishra, C. Därmann, and K. Lücke, "On the development of the Goss texture in iron-3\% silicon," Acta Metall. 32, 2185-2201 (1984).

${ }^{4}$ K. I. Arai and K. Ishiyama, "Rolled texture and magnetic properties of $3 \%$ silicon steel," J. Appl. Phys. 64, 5352 (1988)

${ }^{5}$ A. Hubert and R. Schaefer, Magnetic Domains (Springer, 1998), ISBN: 3540-64108-4.

${ }^{6} \mathrm{C}$. Holt and J. A. Robey, "The AC magnetostriction of $3.25 \%$ grainoriented silicon-iron under combined longitudinal and normal compressive stress," IEEE Trans. Magn. 5, 384-388 (1969).

${ }^{7}$ E. Beyer, L. Lahn, C. Scheepers, and T. Stucky, "The influence of compressive stress applied by hard coatings on the power loss of grain oriented electrical steel sheet," J. Magn. Magn. Mater. 323, 1985-1991 (2011).

${ }^{8}$ W. D. Corner and J. J. Mason, "The effect of stress on the domain structure of Goss textured silicon-iron," Brit. J. Appl. Phys. 15, 709-718 (1964).
${ }^{9}$ A. J. Moses, "Effects of applied stress on the magnetic properties of high permeability silicon-iron," IEEE Trans. Magn. 15, 1575-1579 (1979).

${ }^{10}$ International standard, U.S. patent IEC 60404-2:2008 (28 March 1996).

${ }^{11}$ R. Schäfer and S. Schinnerling, "Bulk domain analysis in FeSi-crystals," J. Magn. Magn. Matter 215-216, 140-146 (2000).

${ }^{12}$ C. Grünzweig, F. Pfeiffer, O. Bunk, T. Donath, G. Kühne, G. Frei, M. Dierolf, and C. David, "Design, fabrication, and characterization of diffraction gratings for neutron phase contrast imaging," Rev. Sci. Instrum. 79, 053703 (2008).

${ }^{13}$ C. Grünzweig, C. David, O. Bunk, M. Dierolf, G. Frei, G. Kühne, R. Schäfer, S. Pofahl, H. M. R. Ronnow, and F. Pfeiffer, "Bulk magnetic domain structures visualized by neutron dark-field imaging," Appl. Phys. Lett. 93, 112504 (2008).

${ }^{14}$ C. Grünzweig, C. David, O. Bunk, M. Dierolf, G. Frei, G. Kühne, J. Kohlbrecher, R. Schäfer, P. Lejec, H. M. R. Ronnow, and F. Pfeiffer, "Neutron decoherence imaging for visualizing bulk magnetic domain structures," Phys. Rev. Lett. 101, 025504 (2008).

${ }^{15}$ C. Grünzweig, C. David, O. Bunk, J. Kohlbrecher, E. Lehmann, Y. W. Lai, R. Schäfer, S. Roth, P. Lejcek, J. Kopecek, and F. Pfeiffer, "Visualizing the propagation of volume magnetization in bulk ferromagnetic materials by neutron grating interferometry," J. Appl. Phys. 107, 09D308 (2010).

${ }^{16}$ I. Manke, N. Kardjilov, R. Schäfer, A. Hilger, M. Strobl, M. Dawson, C. Grünzweig, G. Behr, M. Hentschel, C. David, A. Kupsch, A. Lange, and J. Banhart., "Three-dimensional imaging of magnetic domains," Nat. Commun. 1, 125 (2010).

${ }^{17}$ S. W. Lee, D. Hussey, D. L. Jacobson, C. M. Sim, and M. Arif, "Development of the grating phase neutron interferometer at a monochromatic beam line," Proceedings of ITMNR 08 (2009), Vol. 605, pp. 16-20.

${ }^{18}$ S. W. Lee, Y. K. Jun, and O. Y. Kwon, "A neutron dark-field imaging experiment with a neutron grating, interferometer at a thermal neutron beam line at HANARO," J. Korean Phys. Soc. 58, 730 (2011).

${ }^{19}$ E. Calzada, F. Gruenauer, M. Mühlbauer, B. Schillinger, and M. Schulz, "New design for the ANTARES-II facility for neutron imaging at FRM II," Proceedings of ITNMR 08 (2009), Vol. 605, pp. 50-53.

${ }^{20}$ A. Kaestner, S. Hartmann, G. Kühne, G. Freib, C. Grünzweig, L. Josic, F. Schmid, and E. H. Lehmann, "The ICON beamline - A facility for cold neutron imaging at SINQ," Nucl. Instrum. Methods 659, 387-393 (2011).

${ }^{21}$ H. F. Talbot, "Facts relating to optical science," Philos. Mag. 9, 401-407 (1836).

${ }^{22}$ C. Gruenzweig, G. Frei, E. Lehmann, G. Kühne, and C. David, "Highly absorbing gadolinium test device to characterize the performance of neutron imaging detector systems," Rev. Sci. Instrum. 78, 053708 (2007).

${ }^{23}$ The coating was removed by chemical etching using hydrochloric acid diluted with water.

${ }^{24}$ See http://www.aksteel.com/pdf/markets_products/electrical/AK\%20 CARLITE\%20Lite\%20042413.pdf for further information about mechanical properties of HPSL. 